\title{
INHERITANCE OF ACQUIRED CHARACTERS
}

\begin{abstract}
$\mathrm{HE}$ problem of the inheritance of acquired characters is one that has exercised the minds of biologists since the days of Lamarck. 'That interest in this aspect of evolution is unabated was shown by the very full attendance at the joint discussion of the subject, between the Linnean Society and the Systematics Association, held in the rooms of the Linnean Society on November 1. The discussion was opened by Prof. H. Graham Cannon, who eited Leonard Doncaster's definition of an acquired character as " $\mathrm{a}$ feature developed during the life of the individual possessing it, in response to the action of use or environment". The added qualification that, "as a rule", such acquired characters were adaptive, has led to much of the trouble arising out of attempts to prove or disprove the proposition. There are two types of experiment, depending on the nature of the change in the environment to which the organism is subjected, whether normal, as occurring in Nature, or not. To the neo-Mendelian the distinction does not matter, for as Carter said, "It is easy to imagine a gene doing anything we want it to do". Every organism in equilibrium with its environment must change somehow if external conditions change. The difficulty is to force it to change obviously, and, as MacBride said, if a change from one environment to another causes a change in structure in one generation, a change in the opposite direction should reverse it in an equal time. This is true of adaptive acquired characters. If the acquired character is induced by some abnormal circumstance, it will be an induced mutation and therefore inherited in the normal Mendelian manner. It seems, therefore, that attempts to demonstrate experimentally the inheritance of acquired characters are a waste of time.
\end{abstract}

It is Lamarck's fourth law that states explicitly that acquired characters are inherited; but this law is redundant as it is covered by his second and third laws. To Lamarck, it was not a question of a new character appearing in one generation and being handed on to the next. In accordance with his second law, it would appear in any event, because it was required. The present neo-Mendelian theory is based on the idea that, "New hereditary changes occur spontaneously and at random: they are not guided so as to fit the environment". This view was repudiated by Graham Cannon. Mendelian inheritance can go back only as far as the first Metazoa, since it depends on the orderly behaviour of chromosomes. Below that level, there is no true mitosis or meiosis, on which proof of the Mendelian law is based, and, at the best, only simulacra of these processes in the Protista. The earliest living matter was an amorphous protoplasm, a chemical mixture in equilibrium with its surroundings and, as such, must have reacted according to the physico-chemical law of Le Chatelier. The result would accord with I,amarck's second law. Protoplasm was able to adjust itself in the optimum manner to changes in its surroundings, and it was unlikely that when cells, sex and chromosomes appeared, this quality disappeared. This homœostatic property of protoplasm applied to the morphology as well as the physiology of the organism. It is the main motive force of evolution.

The problem of inheritance in bacteria was discussed by Dr. A. C. R. Dean. In such micro-organisms, the proportions of enzymes in the cell must attain stable ratios after long-continued growth in a given medium. When the medium is changed, different ratios become necessary and the adjustments are made during the lag phase before growth recommences in the new medium. Considerable growth in the new medium is required, before the ratios settle down again to a new stable value. In bacteria, growth and function are closely linked and response to changes in the environment is automatic. Another interpretation of the lag phase is that it represents the time taken by fully adapted mutants to multiply. Experiments with Bact. lactis aerogenes and $\mathrm{D}$ arabinose, designed to test these alternatives, favoured the adaptation hypothesis. 'Thus, there is an increase of at least $\mathbf{4 0}$ per cent in cell mass before cell number increases. No stably adapted strains were obtained by plating out individual bacteria from cultures starting to grow in the new medium. Other tests, with different organisms, indicated that the majority of the cells in a culture undergo adaptation, whether the medium contained a new food material or a drug such as proflavine, streptomycin or chloramphenicol. The stability of an adapted strain depends on how well the training has been impressed on the cells and never seems to become absolute. Even after a thousand generations on drug media, the adaptation, although persisting in some cases for as long as six hundred generations on drug-free media, was eventually lost. The interpretation to be placed on these results is debatable. Dr. Dean inclined to the view that such adaptations represented the development of an existing function, rather than the acquisition of a new one. It is very doubtful to what extent they throw light on the position in multicellular forms.

In attempting to link up the situation in unicellular organisms with that in the higher plants and animals, Dr. R. Melville suggested that the initial changes in the cell were similar in all groups. They depended on the exploitation of existing enzyme systems and alternative metabolic paths. The first result in the higher groups was a phenotypic response, which was not inherited. Environmental changes may set up relentless pressures, and failure to adapt may mean extinction. The probable course of events was that after a variable number of generations, some essential molecule or molecules become attached to a protein molecule, possibly ribose nucleic acid. This may be contained in a cytoplasmic granule and have the properties of a plasmagene. It may pass from cell to cell and be carried over sporadically during fertilization. Afterwards, the plasmagene may be incorporated in a gene and pass into the nuclear mechanism for Mendelian inheritance.

The most critical stage in the suggested adaptive process was probably that leading to the formation of a transmissible particle. As evidence for the occurrence of this process, Waddington's work on the production of pheno-copies for cross-veinless, by 
subjecting puparia of Drosophila melanogaster to a temperature shock, was cited. Individuals inheriting the character were found from the twelfth generation onwards. In plants, it appeared from Darlington's work on male sterility in Epilobium luteum $\times$ hirsutum that plasmagenes restoring fertility appeared after fourteen generations. From knowledge of the physiology of pollen sterility in Frorsythia and other plants, boron may be an important factor in the fertility mechanism. This linked up with the mineral content of the soil, as an example of an environmental factor that may exert an adaptation pressure. In discussing təmpərature, Crosby's paper on the "Role of Plasmogenes in Acquired Adaptations" was mentioned, and it was pointed out that no special assumptions were necessary if the applicability of the Le Chatelier principle were admitted. The effects of temperature were stated more precisely by the van't Hoff principle. It followed, that if a process was controlled partly by exothermic and partly by endothermic reactions, any change of temperature would have a differential effect. Applied to morphology, this could affect the shape of leaves or other organs. An explanation of the topocline in leaf shape in Ulmus coritana was possible, on these lines.

In the unavoidable absence of Prof. Waddington, his papər was read by Dr. A. J. Cain, who said that although random mutation and natural selection, together, constitute a mechanism or generating states of high a priori improbability, many people were not satisfied that this conventional theory contains the whole truth. The existence of environmentally adapted populations within a species could not be wholly irrelevant to the process of evolution. On general grounds, one would expect the capacity of an organism to respond to environmental stress to be under genetic control. Individuals within a population will vary in this respect and natural selection will favour those in which the response is greatest. Conditions for producing, experimentally, a really adaptive change in an organism had not been devised, but artificial selection could be applied to other environmentally induced responses. When Drosophila puparia are subjected to a hot shock, some individuals respond by developing abnormalities in the wing venation. After twelve generations, cross-veinless flies began to appear in untreated individuals. When these were bred together, without further treatment, strains could rapidly be built up, in which nearly all the individuals were cross-veinless. The original phenotypic response was now genetically fixed. Similar results were obtained with populations from different sources. In most wild-type stocks submitted to temperature shock, four types of abnormality occurred. If selection is practised on any one of these, its frequency increases, while that of the others does not. In generalizing from these experiments, it seems possible for a response, that was at first only imperfectly adaptive, to be 'tuned' more closely to the requirements of the animal's life. The mechanism revealed operates in a perfectly 'orthodox' Mendelian way. The most important question remaining for discussion is how it comes about that so many responses of animals to environmental stresses are adaptive. The answer may be based on two considerations. First, that development must be epigenetic, involving interactions between parts, so that, for example, changes in the skeleton are associated with changes in musculature, and vice versa ; secondly, that selection acting on a macroevolutionary time-scale will tend to favour types of epigenetic interaction that offer the possibility of adaptive response to stresses.

In the general discussion following, Dr. W. B. Turrill criticized Prof. Graham Cannon for his statements that bacteria had "no nucleus, no chromosomes and no sexuality". Dr. M. L. R. Petterson directed attention to the evidence, published in recent text-books, for the existence of genes in both bacteria and viruses, and Mr. P. F. Mattingly mentioned a very beautiful experimental demonstration by certain French workers of the existence of a linear arrangement of hereditary factors in Escherichia coli. Prof. Graham Cannon questioned the validity of work with bacteria kept in culture for considerable periods, and suggested that infection by viruses might affect the results. Dr. Dean admitted that the stocks employed had been in culture some years, but their behaviour was always tested in a standard medium before and after experiments with drugs or alternative carbon sources. Dr. S. M. Manton regretted that the discussion had centred so much around single isolated characters. Many characters are intimately correlated for the harmonious working of a whole animal. For example, the control of movement in a centipede involved nearly all parts of the body, and a change in a single character would be ineffective. The numbər of simultaneous gene changes necessary to account for tergite heteronomy in the centipedes is so prodigious that the concept needs reconsideration.

R. Melville

\section{OBITUARY}

Sir Gerald Lenox-Conyngham, F.R.S.

THe working life of Colonel Sir Gerald Ponsonby Lenox-Conyngham, who was born at Moneymore, Londonderry, on August 21, 1866, the fifth son of Sir William Lenox-Conyngham, and educated at the Edinburgh Academy and the Royal Military Academy, Woolwich, may be reckoned from the time of his being commissioned second lieutenant, Royal Engineers, at the early age of nineteen. Even after his ultimate retirement sixty-two years later, he continued, until his death on October 27, to be the valued counsellor of his friends. The first half of his long career was in India ; the remainder in Cambridge. Throughout he was sustained by Lady
Lenox-Conyngham, whom he married in 1890. She was daughter of Surgeon-General Sir A. E. Bradshaw, and there was one daughter of the marriage.

An orderly line of progress throughout may be perceived. Lenox-Conyngham joined the Survey of India in 1889 and was then engaged on astronomical fieldwork for the next dozen years. During 1894-96, as partner to Burrard (later Sir Sidney Burrard), his senior by five years, he was on deputation out of India conducting observations for the precise longitude of India, then embarrassingly in doubt. These two great men-for they were of great stature and of equally notable ability-became and continued fast friends; but they ever remained to each other just ' $B$ ' and ' $C$ '. 